\section{Characterization of Polyphenolics in Grape Pomace Extracts Using ESI Q-TOF MS/MS}

\author{
Zhang S and Zhu MJ* \\ School of Food Science, Washington State University, Pullman, WA, USA
}

\begin{abstract}
Background: Grape pomaces are rich sources of polyphenolics that are reportedly beneficial for health, but their content and quality between red and white pomaces have not been systemically compared.

Methods and findings: The current study compared polyphenolics from different grape extracts and further characterized A-type Proanthocyanidins (PAC), which have not been studied previously. The total contents of polyphenolics, flavonoids and PAC and total antioxidant activities of Red Grape Pomace Extract (RGPE) were higher than those in White Grape Pomace Extract (WGPE). Using direct-infusion electrospray ionization tandem mass spectrometry, glucosides of quercetin and peonidin were detected in both RGPE and WGPE, while quercetin, malvidin derivatives and petunidin 3-p-coumaroylgluside only found in RGPE. (epi)catechins, B-type PACs, A-type PAC dimers, and single A-type linked PAC trimers and tetramers were detected in RGPE, WGPE and Grape Seed Extract (GSE). Other singly and doubly charged A-type PACs only detected in GSE. Furthermore, monogalloylated A-type dimers with (epi)cat and (epi)afz were detected in both GSE and WGPE.

Conclusion: RGPE contains relatively greater amounts of polyphenolics than WGPE, and more A-type PAC was detected in GSE. The total antioxidant content was higher in RGPE than WGPE. Keywords: A type; Antioxidant; Grape pomace; Polyphenolic; Proanthocyanidins; Tandem mass spectrometry
\end{abstract}

\section{Introduction}

Grape pomace is the major byproduct of the wine and juice industry, which is rich in polyphenols including flavonoids (anthocyanins, flavanols, flavonols, and flavanones) and non-flavonoids (phenolic acids and their derivatives, stilbenes, and

*Corresponding author: Zhu MJ, School of Food Science, Washington State University, Pullman, WA 99164, USA, Tel: +1 5093354016; Fax: +1 5093354815 E-mail: meijun.zhu@wsu.edu

Citation: Zhang S, Zhu MJ (2015) Characterization of Polyphenolics in Grape Pomace Extracts Using ESI Q-TOF MS/MS. J Food Sci Nutr 1: 001.

Received: August 04, 2014; Accepted: March 06, 2015; Published: March 20 2015 lignans) [1]. Grape Seed Extracts (GSE) are known for their anti-oxidative and anti-inflammatory effects, and exert various physiological benefits including anti-carcinogenic, anti-aging, anti-diabetic, and cardioprotective effects [2]. Recent studies also show their roles in regulation of intestinal barrier and prevention of intestinal inflammatory diseases [3,4]. However, polyphenolic content and composition of GSE, red and white grape pomace have not been systemically compared, and these contents also differ due to grape cultivar varieties, environmental conditions and geological locations where grapes are produced; as a result, the efficacy of extracts in preventing diseases and protecting health varies. Characterization of polyphenols is not only critical for the quality control of extracts, but also mechanistic studies exploring their biological efficacy.

Flavan-3-ols are the main polyphenolics in grapes, which in general are monomeric catechin (cat) and epicatechin (epicat) and their oligomeric and polymeric (epi)cat known as Proanthocyanidins (PACs) ( $\mathrm{n} \leq 5$, oligomers and $\mathrm{n}>5$, polymers). Proanthocyanidins have two types of linkages: B-type has only single linkage of C4-C6' or C4-C8' between (epi)cat units while A-type has double linkages between C4-C8' and O7'-C2. In general, B-type PACs consist of only B-type linkages, while A-type PACs have A-type linkages in addition to the B-type bonds [5]. Currently, the A-type PACs and their derivatives have not been well-characterized in grapes and their products [6,7], which leads to an important knowledge gap, considering A-type and B-type PACs may have different bioactivity. Indeed, an A-type PAC dimer from cranberry was more effective than those enriched in B-types in inhibiting in vitro bacterial adherence [8]. Furthermore, PACs rich in A-type linkages were more effective in the inhibition of pancreatic lipase activity than that in B-types [9]. In this study, we characterized polyphenols in Red and White Grape Pomace Extracts (RGPE and WGPE), further characterized and compared the main compounds among RGPE, WGPE and commercial GSE especially A-type PACs using direct infusion Electrospray Ionization (ESI) tandem mass spectrometry.

\section{Materials and Methods}

\section{Grape pomaces and chemicals}

Red and white grape pomace mainly containing grape skins, seeds as well as some stems were generously provided by Woodward canyon winery (Lowden, WA). The red pomace was generated from Cabernet Sauvignon and the white was from Chardonnay. Both red and white grape pomace were freeze-dried and ground to 40-60 mesh powders. GSE (GravinolSuper ${ }^{\mathrm{TM}}$ ) was purchased from OptiPureChemco Industries Inc. (Los Angeles, CA).

Ethanol, catechin, rutin, gallic acid, formic acid, glacial acetic acid, vanillin, aluminum chloride, DPPH• (2,2-diphenyl-1-picrylhydrazyl) radical, and Folin-Ciocalteu's reagent were purchased from Sigma-Aldrich Co. (St. Louis, MO, USA). Sodium carbonate and sodium acetate were from JT Baker (Center Valley, PA, USA).

\section{Sample preparation}

After testing the efficiency of extraction by using different extraction durations (5 to $30 \mathrm{~min}$ ) and temperatures $\left(40\right.$ and $50^{\circ} \mathrm{C}$ ), 
with and without acidified solvents, in combination with or without ultrasonic treatment (Ultrasonic cleaner XSPS-180-6L, SharperTek, Pontiac, MI, USA), we selected $80 \%$ EtOH-1\% Formic Acid (FA) in combination with ultrasonic extraction for $15 \mathrm{~min}$ at $40^{\circ} \mathrm{C}$ for extracting polyphenols from grape pomace. Briefly, pomace powders were first treated with hexane to remove non-polar compounds such as lipids at room temperature. $80 \% \mathrm{EtOH}-1 \% \mathrm{FA}$ was added into dried hexane extracted grape pomace powder at 10:1 ratio of solvent to sample (volume/weight). The sample was vortexed and incubated at room temperature for one hour, followed by $15 \mathrm{~min}$ ultrasonic treatment $\left(40^{\circ} \mathrm{C}\right)$, then centrifuged at $12,000 \mathrm{rpm}$ for $15 \mathrm{~min}$. The supernatants containing extracted polyphenols were collected. The residues were re-extracted twice. Supernatants from three extractions were combined and kept at $-20^{\circ} \mathrm{C}$ till analyses.

\section{Chemical composition and antioxidant activity of Grape Pomace Extracts (GPEs)}

All analyses were performed in 96-well microplates using Synergy H1 Hybrid microplate reader (BioTek Instruments Inc., Winooski, VE, USA). All reactions were conducted at room temperature, and incubation time for specific reaction was determined by its kinetics with the desired wavelength.

Total phenolic content: Total phenolic content was determined using the modified Folin-Ciocalteu procedure [10]. Gallic acid (1.0 to 50.0 $\mu \mathrm{g} / \mathrm{ml}$ ) was used to generate the standard curve. In brief, $200 \mu \mathrm{L}$ of diluted GPEs and standard solutions were added into each well, followed by $12.5 \mu \mathrm{L}$ of Folin-Ciocalteu's reagent and then $37.5 \mu \mathrm{L}$ of $20 \%$ $\mathrm{Na}_{2} \mathrm{CO}_{3}$. The absorbance at $760 \mathrm{~nm}$ was read after 2 hour incubation. The results were expressed as milligrams of gallic acid equivalent per gram of dried pomace weight (mg GAE/g DW).

Total flavonoid content: The modified $\mathrm{AlCl}_{3}$-acetate method [11] was used to measure total flavonoid content. Briefly, $50 \mu \mathrm{L}$ of diluted GPEs and standard solutions were mixed with $150 \mu \mathrm{L}$ of $5 \% \mathrm{AlCl}_{3}$ and 50 $\mu \mathrm{L}$ of acetate buffer ( $\mathrm{pH}$ 5.0) sequentially. The absorbance at $420 \mathrm{~nm}$ was measured after one hour incubation at room temperature. Rutin was used as the standard with a range of 4.0 to $148.0 \mu \mathrm{g} / \mathrm{ml}$ and total flavonoid content was expressed as milligrams of rutin equivalent per gram of dried pomace weight (mg RE/g DW).

Total PAC content: Total PAC content was analyzed by the modified Vanillin- $\mathrm{HCl}$ procedure [12]. In brief, $50 \mu \mathrm{L}$ of diluted GPEs and standard solutions were mixed with $150 \mu \mathrm{L}$ of $4 \%$ vanillin and $50 \mu \mathrm{L}$ of concentrated hydrochloride. The absorbance was measured at $500 \mathrm{~nm}$ after incubation for $30 \mathrm{~min}$. The total PAC content was determined using catechin as a standard ranging between 5.0 and $250.0 \mu \mathrm{g} / \mathrm{ml}$. Data were expressed as milligrams of catechin equivalent per gram of dried pomace weight (mg CE/g DW).

DPPH radical scavenging assay: The total antioxidant activity in GPEs was determined by the ability to scavenge DPPH • [13]. 200 $\mu \mathrm{L}$ of $\mathrm{DPPH} \bullet$ solution $\left(6 \times 10^{-5} \mathrm{M}\right)$ were added into microplate wells containing $50 \mu \mathrm{L}$ of diluted GPEs or standards. The DPPH. scavenging activity was measured at $515 \mathrm{~nm}$ after $90 \mathrm{~min}$ incubation at room temperature. Data were expressed as $\mathrm{mg} \mathrm{CE} / \mathrm{g}$ DW that was calculated by the standard curve of catechin in the range of 0.3 to $6.0 \mu \mathrm{g} / \mathrm{mL}$. The percent inhibition of DPPH• radical scavenging activity was calculated by the following equation: inhibition (\%) $=\frac{A_{\text {control }}-A_{\text {sample }}}{A_{\text {control }}} \times 100$, where $A$ is the absorbance.

\section{Characterization of grape extracts using ESI Q-TOF-MS/ MS}

The mass spectra were collected via the direct infusion on a Waters ESI Q-TOF Premier (Waters, USA) with electrospray ionization source equipped with MassLynxv4.1. Both positive and negative ion ESI mode MS/MS analyses were performed under the following conditions: the capillary voltage, $+3.5 \mathrm{KV} /-3.0 \mathrm{KV}$ [ESI+/ESI-]; the source temperature, $110^{\circ} \mathrm{C}$; the sample cone, $30 \mathrm{~V} / 40 \mathrm{~V}$ [ESI+/ESI-]; the desolvation (L/hr), 300/350 [ESI+/ESI-]; the scan range, 90-2190 amu; the scan rate, $1 \mathrm{sec} / \mathrm{scan}$. Samples were diluted in $25 \%$ methanol with $0.5 \%$ formic acid and directly infused into the electrospray source at the flow-rate of $3 \mu \mathrm{L} / \mathrm{min}$. The $\mathrm{m} / \mathrm{z}$ number of precursor ion marked with ${ }^{\star}$ indicates that precursor ion generated from ESI+ mode.

The relative percentages of (epi)cat and PACs were calculated from their respective peak intensities divided by total peak intensities of (epi)cat and PACs including galloylated PACs.

\section{Statistical analysis}

All data were presented as mean with their corresponding standard deviations from three independent experiments. The student's t-test was used to identify difference. Differences at $p \leq 0.05$ were considered significant.

\section{Results and Discussion}

\section{Physical properties of grape pomace}

The extraction yield (dried weight of crude extract/dried weight of pomace $\mathrm{x} 100$ ) for the dried red and white grape pomaces was 30.65 $\pm 0.95 \%$ and $54.76 \pm 0.18 \%$, respectively. The extraction yield of white grape pomace is much higher than previous reported [14,15] while the red grape pomace is similar to others [16].

\section{Polyphenolics in grape pomace extracts}

The total phenolic, flavonoid and PAC contents are presented in table 1 . The total phenolic and flavonoid contents were higher in RGPE than those in WGPE. The total flavonoid contents in RGPE and WGPE accounted for about $63 \%$ and $25 \%$ of total phenolic content, respectively. The total phenolic level in both RGPE and WGPE was higher than the value reported previously using the same extraction solvent (30.4 for Cabernet Franc and 24.5 for Chardonnay) [17]. Our data are consistent with the results obtained from Tinta Cao (red) and Chardonnay (white) [18], Cabernet sauvignon, Pinot Noir and Merlot (red) [16], and white grapes cultivated in Turkey [19]. Furthermore, the total phenolic content in WGPE was largely consistent with the content identified in four white grape cultivars (30.9-46.5 mg GAE/g DW) [14]. The difference in phenolic contents by different reports is likely due to the variation in grape cultivars, climate and culture conditions, as well as extraction methods $[17,18]$. The total PAC content is higher in RGPE than that in WGPE (Table 1), in agreement with a previous report [14].

\section{Total antioxidant activities}

In agreement with their higher content of total phenolics, flavonoids and PACs, the RGPE had higher DPPH• free radical scavenging capacity and greater antioxidant activity than WGPE (Table 1). 


\begin{tabular}{|l|c|c|}
\hline & RGPE & WGPE \\
\hline Total phenolics (mg GAE/g DW) & $69.83 \pm 4.53$ & $58.15 \pm 5.21^{*}$ \\
\hline Total flavonoids (mg RE/g DW) & $43.89 \pm 1.22$ & $14.32 \pm 1.67^{*}$ \\
\hline Total Proanthocyanidins (mg CE/g DW) & $133.79 \pm 6.74$ & $92.10 \pm 6.00^{*}$ \\
\hline Antioxidant activity (mg CE/g DW) & $74.48 \pm 1.12$ & $58.66 \pm 1.92^{*}$ \\
\hline DPPH• inhibition (\%) & $68.28 \pm 0.52$ & $62.74 \pm 1.34^{*}$ \\
\hline
\end{tabular}

Table 1: Total phenolics, flavonoids and proanthocyanidins and total antioxidant activities of grape pomace extracts.

RGPE: Red Grape Pomace Extract; WGPE: White Grape Pomace Extract; Total content of phenolics, flavonoids and Proanthocyanidins (PACs) is expressed respectively as $\mathrm{mg}$ of Gallic Acid Equivalent (GAE), mg of Rutin Equivalent (RE) and $\mathrm{mg}$ of Catechin Equivalent (CE) per gram of Dried Weight (DW) Total antioxidant activity is determined with the DPPH radical scavenging activity and expressed as $\mathrm{mg}$ of Catechin Equivalent (CE) per gram of dried weight. Data are means of three independent experiments. Data were presented as Mean \pm SEM; *: $p<0.05$

\section{TOF-MS profiles and main components of RGPE, WGPE and GSE}

Table 2 lists the phenolic compounds putatively identified by direct infusion tandem MS in both negative and positive modes. Figure 1 shows the direct infusion ESI Q-TOF mass spectra of RGPE, WGPE and GSE in both positive and negative ion modes. Inserts in figure $2 \mathrm{~A}$, are enlarged spectra in negative mode showing overlapped isotope patterns of PAC dimers to hexamers and doubly charged tetramers and monogalloylated heptamers and nonamers containing A-and B-types. The PACs were further examined with their fragments processing through main fragmentation patterns of Retro-Diels-Alder (RDA) fission, Heterocyclic Ring Forming fission (HRF), and Quinonemethide (QM) fission as demonstrated in figure 3, as well as Benzofuran Forming (BFF) fission [5,20,21].

\section{Organic acids, (epi)catechins and anthocyanins}

At ESI-, gallic acid ([M-H] $]^{-}$ion at $\left.\mathrm{m} / \mathrm{z} 169\right)$ was presented in all grape extracts and was confirmed by its MS/MS fragment at m/z 125 . The $[\mathrm{M}-\mathrm{H}]$ ] ions of $\mathrm{m} / \mathrm{z} 133,149$ and 191 were detected in both RGPE and WGPE while the $[\mathrm{M}-\mathrm{H}]^{-}$at $\mathrm{m} / \mathrm{z} 179$ was presented only in WGPE (Table 2); they are malic acid, tartaric acid, citric acid and caffeic acid, respectively (Figure $1 \mathrm{~A}, \mathrm{~B})[22,23]$.

The $[\mathrm{M}-\mathrm{H}]^{-}$ion at $\mathrm{m} / \mathrm{z} 301$ were detected only in RGPE, which likely is quercetin. Its fragment ions mainly are $\mathrm{m} / \mathrm{z} 273$ [M-H-28(CO)], 257 [M-H-44( $\left.\left(\mathrm{CO}_{2}\right)\right]^{-}, 229$ [M-H-44( $\left.\left(\mathrm{CO}_{2}\right)-28(\mathrm{CO})\right]$ (Table 2), which was similar to a previous report [24].

At ESI-, the [M-H] ions at $\mathrm{m} / \mathrm{z} 463$ and 477 were observed in RGPE, WGPE and GSE, which might be quercetin 3-glucoside and quercetin 3-glucuronide with the fragment at $\mathrm{m} / \mathrm{z} 300$ (loss of a glucosyl unit) and 301 (loss of a glucuronate group), respectively (Table 2). Both of them were reported previously in grape skin at ESI- [25].

ESI+ signals attributable to anthocyanins were observed in grape pomace extracts. The $[\mathrm{M}+\mathrm{H}]^{+}$ion at $\mathrm{m} / \mathrm{z} 479^{*}\left({ }^{*}\right.$ stands precursor ions generated from ESI+ mode) was detected in both RGPE and WGPE (Figure $1 \mathrm{C}, \mathrm{D}$ ), which was assigned to petunidin-3-glucoside confirmed by its fragments at $\mathrm{m} / \mathrm{z} 303$ and 317 (Table 2) [26]. The $[\mathrm{M}+\mathrm{H}]^{+}$ions at $\mathrm{m} / \mathrm{z} 463^{*}, 493^{*}, 505^{*}, 535^{*}, 625^{*}, 639^{*}$, and $655^{*}$ could be assigned to peonidin 3-glucoside, malvidin 3-glucoside, peonidin 3-acetylglucoside, malvidin 3-acetylglucoside, petunidin 3 -p-coumaroylglucoside, malvidin 3-p-coumaroylglucoside, and malvidin 3-(6-O-caffeoyl) monoglucoside (Figure $1 \mathrm{C}, \mathrm{D})$ confirmed by their fragments of 301 (loss of $162 \mathrm{Da}$, a glucosyl unit), 331 (loss of $162 \mathrm{Da}$ ), 301 (loss of $204 \mathrm{Da}$, an acetyl glucosyl unit), 331 (loss of $204 \mathrm{Da}$ ), 317 (loss of $308 \mathrm{Da}$, a coumaroylglucosyl unit), 331 (loss of $308 \mathrm{Da}$ ), and 331 (lose of $224 \mathrm{Da}$, a caffeoylglucosyl unit) (Table 2), respectively [26-28]. Of which, peonidin 3-glucoside and peonidin 3-acetylglucoside were detected in both RGPE and WGPE, while malvidin derivatives and petunidin 3-p-coumaroylgluside only found in RGPE (Table 2).

The $[\mathrm{M}-\mathrm{H}]^{-}$ion at $\mathrm{m} / \mathrm{z} 491$ could be assigned to malvidin 3 -glucoside with the fragment at $\mathrm{m} / \mathrm{z} 329$ (loss of a glucosyl unit), which was detected in RGPE and WGPE and was reported previously in grape skin as well [25].

Catechin and epicatechin were found in all grape extracts at both ESI+ and ESI- with the precursor ion at $\mathrm{m} / \mathrm{z} 291^{*}$ and 289 , respectively (Figure 1 and 2) and backed by their characteristic fragmentations (Table 2) mainly via loss of one water for both of them, RDA (loss of $152 \mathrm{Da}$ ), HRF (loss of $126 \mathrm{Da}$ ) and BFF for the $\mathrm{m} / \mathrm{z} 291^{*}$ precursor ion, and loss of a $-\mathrm{CH}_{2}-\mathrm{CHOH}$ group or $\mathrm{CO}_{2}$, loss of $\mathrm{C}_{4} \mathrm{H}_{4} \mathrm{O}_{2}$ from the A-ring and $\mathrm{C}_{6} \mathrm{H}_{6} \mathrm{O}_{2}$ from B-ring for the $\mathrm{m} / \mathrm{z} 289$ to generate corresponding fragments $[20,29,30]$.

Monogalloylated B-type (epi)cat oligomers: The [M-H] ions at m/z 729,1017 and 1305 could be assigned to monogalloylated B-type dimers, trimers and tetramers with 2,3 and 4 possible structures, respectively. They were all detected in RGPE, WGPE and GSE (Figure $1 \mathrm{~A}, \mathrm{~B}$ and $2 \mathrm{~A}$ ) and their main fragments are listed in table 2. The fragments of $[\mathrm{M}-\mathrm{H}]^{-}$ions at $\mathrm{m} / \mathrm{z} 729$ and 1017 have been characterized previously via loss of $152 \mathrm{Da}$ (RDA or galloyl group), loss of $126 \mathrm{Da}$ (HFR at the top unit), loss of water, and QM (upper and lower unit after loss of galloyl group) [7,31-33]; the fragments of $[\mathrm{M}-\mathrm{H}]^{-}$at $\mathrm{m} / \mathrm{z} 1305$ have not been described, which processes the similar fragmentaion patterns of $[\mathrm{M}-\mathrm{H}]$ - ions at m/z 729 and 1017. The $[\mathrm{M}-\mathrm{H}]^{-}$ions assignable to monogalloylated pentamers ( $\left.\mathrm{m} / \mathrm{z} 1593\right)$ and hexamers $(\mathrm{m} / \mathrm{z} 1881)$ were also detected in RGPE and WGPE (data not shown).

Under ESI+ the monogalloylated B-type dimers at $\mathrm{m} / \mathrm{z} 731^{*}$ were detected in RGPE, WGPE and GSE; while the monogalloylated B-type trimers $\left(\mathrm{m} / \mathrm{z} 1019^{*}\right)$ and tetramers $\left(\mathrm{m} / \mathrm{z} 1307^{*}\right)$ were presented only in RGPE and GSE (Table 2). They all have similar fragmentation pattern as those at ESI-.

A-type PACs: A-type PACs were previously reported in other foods such as peanut skins, hops, and raspberry. However, they have been barely reported in grapes and their products $[6,34]$, which were characterized in this study.

The observed $[\mathrm{M}-\mathrm{H}]^{-}$ions at $\mathrm{m} / \mathrm{z} 575,863,1151,1439$, and 1727 revealed a series of compounds with a mass difference of 288 $\mathrm{Da}$ that can be attributed to A-type PAC dimers, trimers, tetramers, pentamers and hexamers, respectively. They displayed 2 amu difference from the corresponding B-type PACs at $\mathrm{m} / \mathrm{z} 577,865,1153$, 1441 and $1729[5,7,24,31,35,36]$. Further, the observed $[\mathrm{M}+\mathrm{H}]^{+}$ions for A-type PAC dimers to hexamers at $\mathrm{m} / \mathrm{z} 577^{\star}, 865^{\star}, 1153^{*}, 1441^{*}$ and $1729^{*}$ also present 2 amu difference from the corresponding B-types at $\mathrm{m} / \mathrm{z} 579^{*}, 867^{*}, 1155^{*}, 1443^{*}$ and $1731^{*}[6,20,21]$ (Figure 1,2 and Table 2).

Figure 3, showed the fragmentation patterns for three selected precursor ions at ESI- mode. Figure $3 \mathrm{~A}$ and $3 \mathrm{~B}$, showed the fragment pathways of A-type (m/z 575) and B-type (m/z 577) dimers. Their characteristic fragmentations are mainly via HRF (loss of $126 \mathrm{Da}$ ), 


\begin{tabular}{|c|c|c|c|c|c|c|}
\hline \multirow{2}{*}{ Compound } & \multicolumn{2}{|c|}{ Precursor ion } & \multirow{2}{*}{ Product lons (MS/MS) } & \multirow{2}{*}{ GSE } & \multirow{2}{*}{ RGPE } & \multirow{2}{*}{ WGPE } \\
\hline & Measured & Calculated & & & & \\
\hline \multicolumn{7}{|l|}{ Organic acids and flavonols } \\
\hline Malic acid & 133.0157 & 133.0137 & & & + & + \\
\hline Tartaric acid & 149.0080 & 149.0086 & & & + & + \\
\hline Gallic acid & 169.0109 & 169.0137 & 125 & + & + & + \\
\hline Caffeic acid & 179.0451 & 179.0344 & 135 & & & + \\
\hline Citric acid & 191.0220 & 191.0192 & & & + & + \\
\hline Quercetin & 301.0332 & 301.0348 & $273,257,229,179,151,137$ & & + & \\
\hline Quercetin 3-glucoside & 463.0952 & 463.0877 & 300,133 & + & + & + \\
\hline Quercetin 3-glucuronide & 477.0767 & 477.0669 & 301,133 & + & + & + \\
\hline \multicolumn{7}{|l|}{ Anthocyanines } \\
\hline Peonidin 3-glucoside & $463.0934^{*}$ & 463.1240 & 300,301 & & + & + \\
\hline Petunidin 3-glucoside & $479.0968^{*}$ & 479.1190 & 303,317 & & + & + \\
\hline \multirow{2}{*}{ Malvidin 3-glucoside } & 491.1352 & 491.1190 & 329,149 & & + & + \\
\hline & $493.1323^{*}$ & 493.1346 & $331,315,287,270,242$ & & + & \\
\hline Peonidin 3-acetylglucoside & $505.1450^{*}$ & 505.1346 & $301,219,145,127$ & & + & + \\
\hline Malvidin 3-acetylglucoside & $535.1390^{*}$ & 535.1454 & $331,315,287,270,242$ & & + & \\
\hline Petunidin 3-p-coumaroylglucoside & $625.1512^{*}$ & 625.1557 & $463,354,317$ & & + & \\
\hline Mavindin 3-p-coumaroylglucoside & $639.1714^{*}$ & 639.1714 & $463,331,315,287,270,242$ & & + & \\
\hline Malvidin 3-(6-O-caffeoyl) monoglucoside & $655.1688^{*}$ & 655.1663 & $381,331,301$ & & + & \\
\hline \multicolumn{7}{|l|}{ Monogalloylated (epi)cat oligomers } \\
\hline \multirow{2}{*}{$\begin{array}{l}\text { Monogalloylated A-type dimers of (epi)cat } \\
\text { and (epi)afz }\end{array}$} & 711.1318 & 711.1350 & $693,559,423,407,289,285,137$ & + & & + \\
\hline & $713.1506^{*}$ & 713.1506 & $695,561,425,409,289,287,139$ & + & & \\
\hline \multirow{2}{*}{ Monogalloylated B-type PAC dimers } & 729.1400 & 729.1456 & $603,577,575,559,441,407,289,169$ & + & + & + \\
\hline & $731.1501^{*}$ & 731.1612 & & + & + & + \\
\hline \multirow{2}{*}{ Monogalloylated B-type PAC trimers } & 1017.2156 & 1017.2089 & $891,865,729,695,577,575,407,289,287$ & + & + & + \\
\hline & $1019.1840^{*}$ & 1019.2246 & $867,731,579,577,441,381,291,289,219$ & + & + & \\
\hline \multirow{2}{*}{ Monogallyolated B-type PAC tetramers } & 1305.2811 & 1305.2723 & $\begin{array}{l}\begin{array}{l}1179,1153,1017,1015,865,863,729,727,577,575, \\
289,287\end{array}\end{array}$ & + & + & + \\
\hline & $1307.2263^{*}$ & 1307.2880 & $\begin{array}{l}1155,1019,1017,867,731,729,579,577,493,381, \\
291,289,219\end{array}$ & + & + & \\
\hline \multicolumn{7}{|l|}{ (epi)catechins and PACs } \\
\hline \multirow{2}{*}{ (epi)catechin } & 289.0698 & 289.0712 & $271,245,205,179,151,137$ & + & + & + \\
\hline & $291.0896^{*}$ & 291.0869 & $273,249,207,169,165,151,147,139,123$ & + & + & + \\
\hline \multirow{2}{*}{ A-type PAC dimers } & 575.1196 & 575.1189 & $539,449,423,407,289,285$ & + & + & + \\
\hline & $577.1323^{*}$ & 577.1345 & $559,437,451,409,425,299,289,287$ & + & + & + \\
\hline \multirow{2}{*}{ B-type PAC dimers } & 577.1385 & 577.1345 & $559,451,425,407,289,287$ & + & + & + \\
\hline & $579.1466^{*}$ & 579.1502 & $561,453,427,409,397,301,291,289,287,275,163$ & + & + & + \\
\hline \multirow[b]{2}{*}{ B-type PAC trimers } & 865.1990 & 865.1979 & $739,713,695,577,575,449,451,425,407,289,287$ & + & + & + \\
\hline & $867.2170^{*}$ & 867.2136 & $\begin{array}{l}715,697,579,577,559,535,495,427,381,291,289, \\
287\end{array}$ & + & + & + \\
\hline \multirow[t]{2}{*}{ B-type PAC tetramers } & 1153.2675 & 1153.2613 & $\begin{array}{l}\text { 1027, } 1001,983,865,863,693,577,575,425,407, \\
289,287\end{array}$ & + & + & + \\
\hline & $1155.2339^{*}$ & 1155.2769 & $1003,867,865,579,577,493,381,291,289,219$ & + & + & + \\
\hline \multirow[b]{2}{*}{ B-type PAC pentamers } & 1441.3232 & 1441.3247 & $1153,1151,865,863,577,575,289,287$ & + & + & + \\
\hline & $1443.2625^{*}$ & 1443.3403 & $\begin{array}{l}\text { 1266, } 1155,1153,867,865,579,577,493,381,291, \\
289,219\end{array}$ & + & + & + \\
\hline \multirow{2}{*}{ B-type PAC hexamers } & 1729.3572 & 1729.3882 & & + & + & + \\
\hline & $1731.2931^{*}$ & 1731.4037 & & + & + & + \\
\hline Double A-type linked PAC trimers & 861.1669 & 861.1666 & $\begin{array}{l}843,735,709,693,691,575,573,571,449,421,411, \\
289,287,285\end{array}$ & + & & \\
\hline & $863.1572^{\star}$ & 863.1823 & & + & & \\
\hline
\end{tabular}


Table 2, continued.

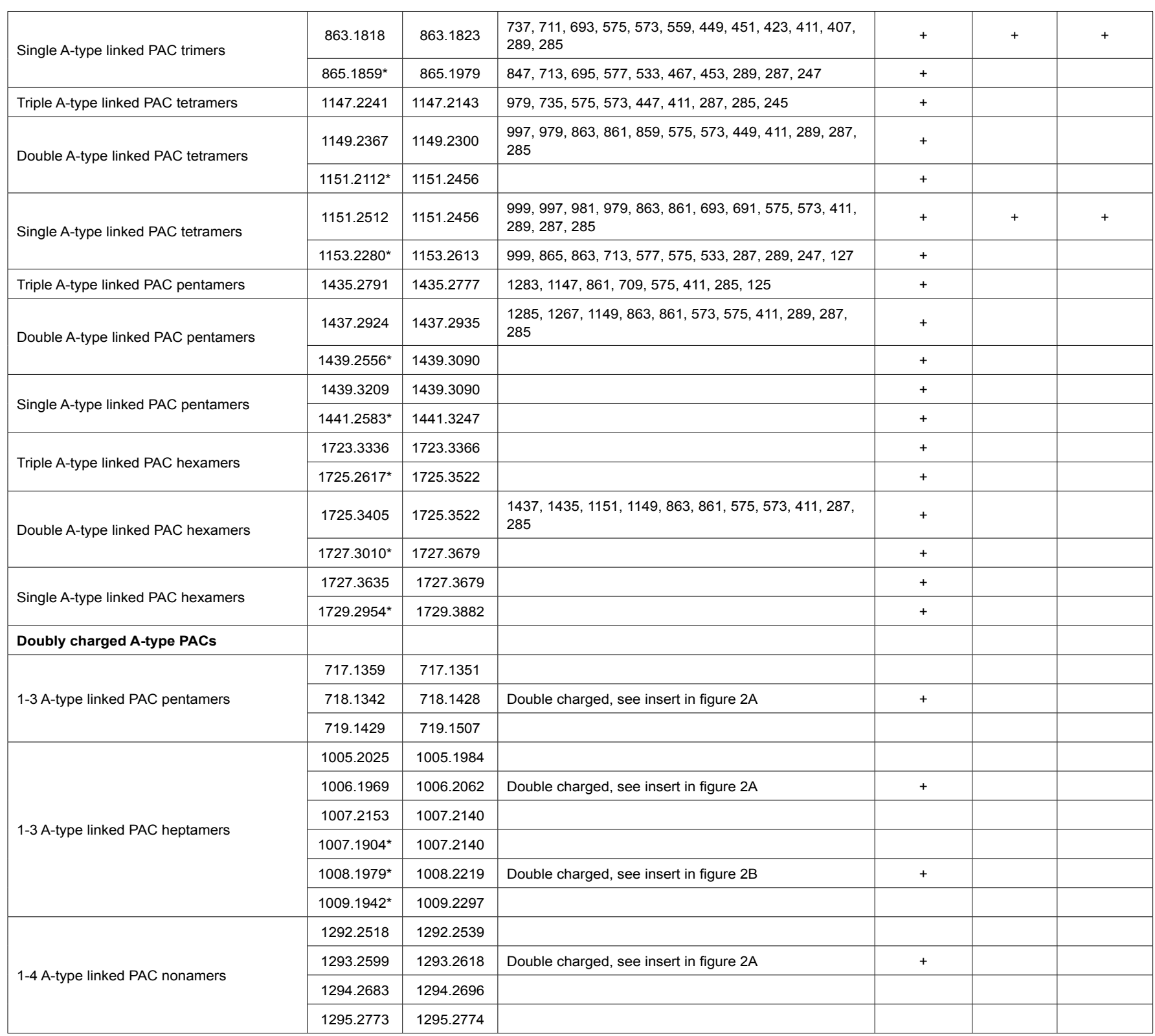

Table 2: Main compounds of grape extracts analyzed by ESI-Q-TOF-MS/MS

GSE: Grape Seed Extract; PAC: Proanthocyanidin; RGPE: Red Grape Pomace Extract; WGPE: White Grape Pomace Extract; Precursor ion marked with * means $[\mathrm{M}+\mathrm{H}]+$, otherwise stands for $[\mathrm{M}-\mathrm{H}]-;+$ : means this compound was detected with reasonable intensity

RDA (loss of $152 \mathrm{Da}$ ) and QM cleavages at the top and bottom units (Table 2), which are consistent with previous reports at ESI- for A-type [5,24,37-39] and for B-type [5,7,29,33,35,39]. At ESI+, the main fragments for both A-type $\left(\mathrm{m} / \mathrm{z} 577^{*}\right)$ and B-type $\left(\mathrm{m} / \mathrm{z} 579^{*}\right)$ dimers (Table 2) are also similar to previous reports [20,21].

One monogalloylated A-type PAC dimer with the $[\mathrm{M}-\mathrm{H}]^{-}$at $\mathrm{m} / \mathrm{z} 711$ was observed in WGPE and GSE, which gave the MS/MS fragments at m/z 693 ([M-H-18] , loss of one water), 559 ([M-H-152] , loss of a galloyl group or via RDA if the terminal unit is (epi)cat), 423 (QM of $\mathrm{m} / \mathrm{z} 711$ or from the fragment ion at $\mathrm{m} / \mathrm{z} 559$ via RDA if the terminal unit is or to be (epi)afz), 407 (from the ion at $\mathrm{m} / \mathrm{z} 559$ via RDA if the terminal unit is or to be (epi)cat), 289 (QM cleavage from terminal unit of (epi)cat while the top unit is (epi)afz after loss of galloyl group and 285 (QM cleavage from terminal unit of (epi)afz while the top unit is (epi)cat after loss of galloyl group), and 137 (RDA of fragment at $\mathrm{m} / \mathrm{z} 289$ ) (Figure $3 \mathrm{C}$ and Table 2). It could be (epi) catG-A-(epi)afz, (epi)afzG-A-(epi)cat, (epi)cat-A-(epi)afzG, or (epi) afz-A-(epi)catG [(epi)afz G, (epi)afzelechin 3-O-gallate].

A-type dimers and B-type PAC dimers to hexamers were detected in all grape extracts under both ESI- and ESI+. In addition, single A-type linked PAC trimers and tetramers can be detected in GSE, RGPE and WGPE under ESI- (Table 2). Otherwise, all A-type PACs described below only detected in GSE under ESI+ and/or ESI(Table 2 and Figure $2 \mathrm{~A}, \mathrm{~B}$ ).

Under ESI- mode, precursor ions at $\mathrm{m} / \mathrm{z} 863$ and 861 could be assigned to PAC trimers with 1 and 2 A-type linkages, respectively (insert in Figure 2A). Their main fragments are aligned with 
Citation: Zhang S, Zhu MJ (2015) Characterization of Polyphenolics in Grape Pomace Extracts Using ESI Q-TOF MS/MS. J Food Sci Nutr 1: 001.

- Page 6 of $10 \bullet$

A

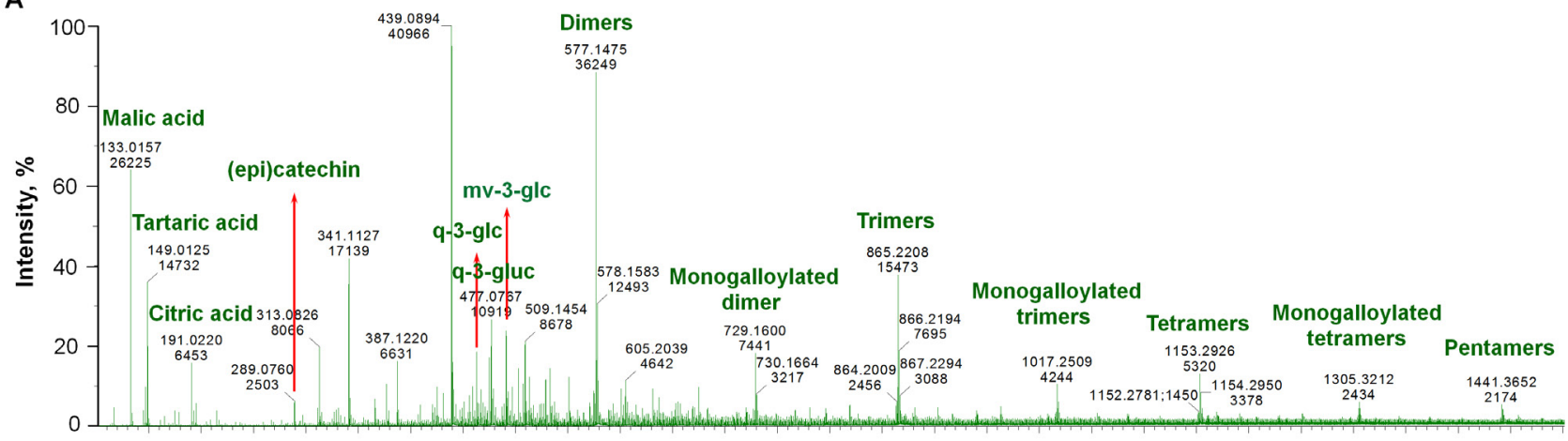

B

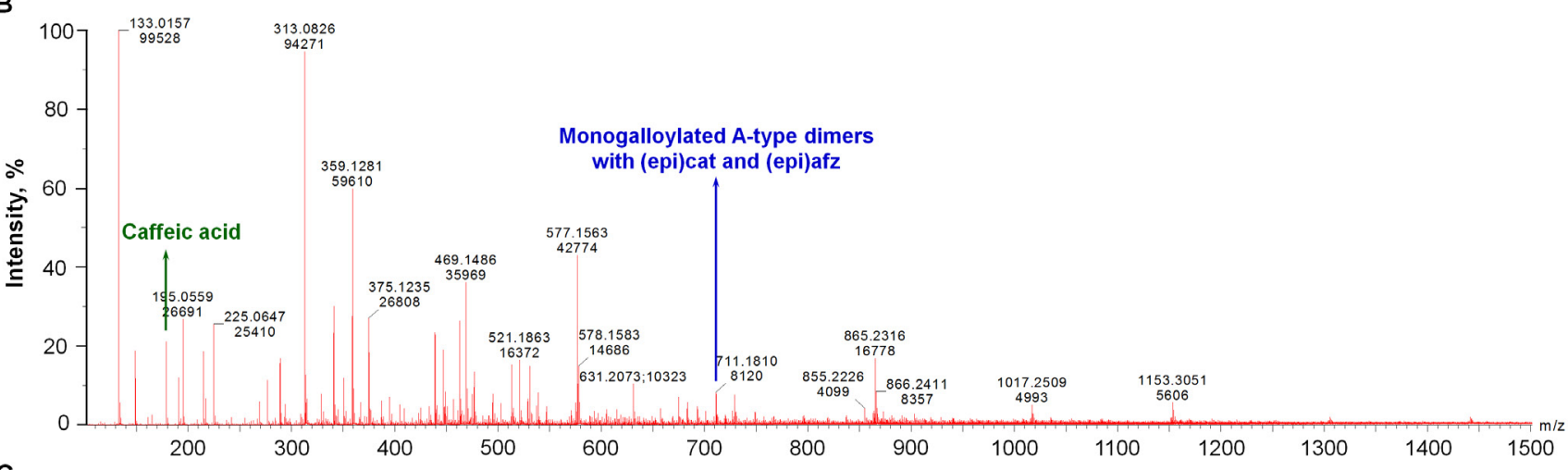

C

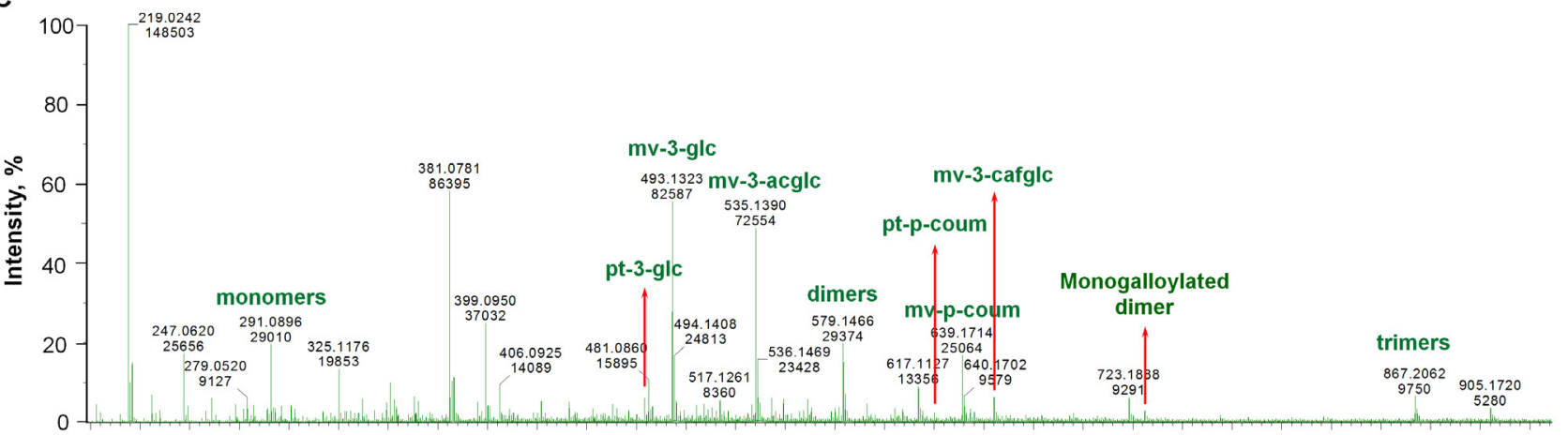

D

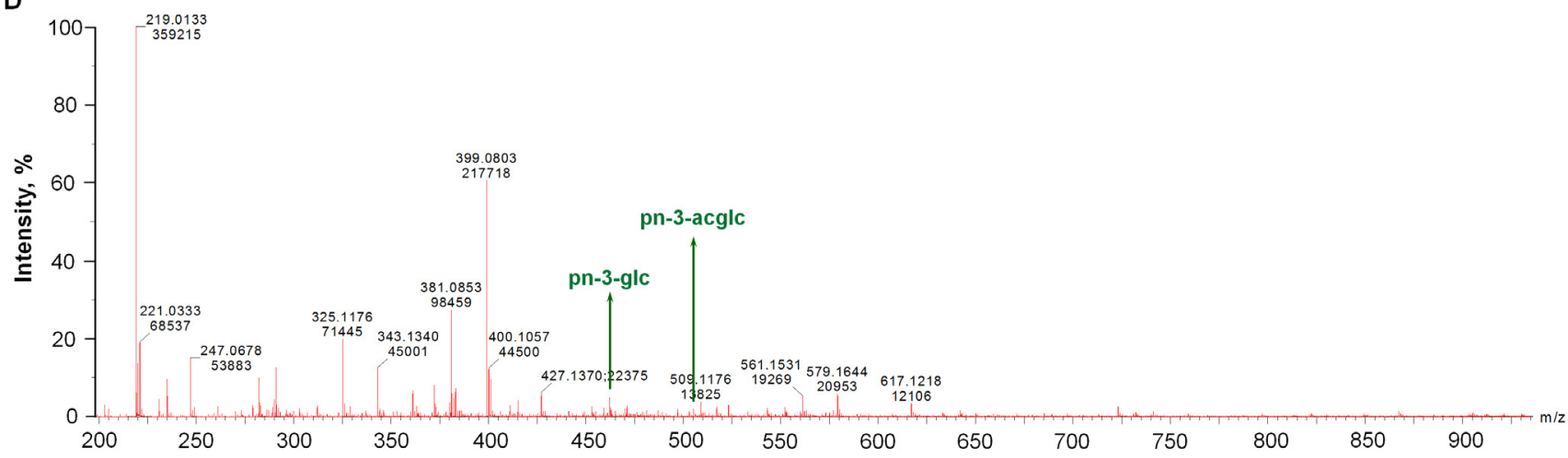

Figure 1: Direct-infusion ESI-Q-TOF-MS profiles of grape pomace extracts at positive and negative ion ESI modes.

A: RGPE, ESI-; B: WGPE, ESI-; C: RGPE, ESI+; D: WGPE, ESI+

mv-3-acglc: malvidin 3-acetylglucoside; mv-3-cafglc: malvidin 3-(6-O-caffeoyl) monoglucoside; mv-3-glc: malvidin 3-glucoside; mv-p-coum: mavindin 3-p-coumaroylglucoside; pt-3-glc: petunidin 3-glucoside; pt-p-coum: petunidin 3-p-coumaroylglucoside; pn-3-glc: peonidin 3-glucoside; pn-3-acglc: peonidin 3-acetylglucoside; q-3-glc: quercetin 3-glucoside; q-3-gluc: quercetin 3-glucuronide

J Food Sci Nutr ISSN: 2470-1076, Open Access Journal

Volume $1 \cdot$ Issue $1 \cdot 100001$

DOI: $10.24966 / F S N-1076 / 100001$ 


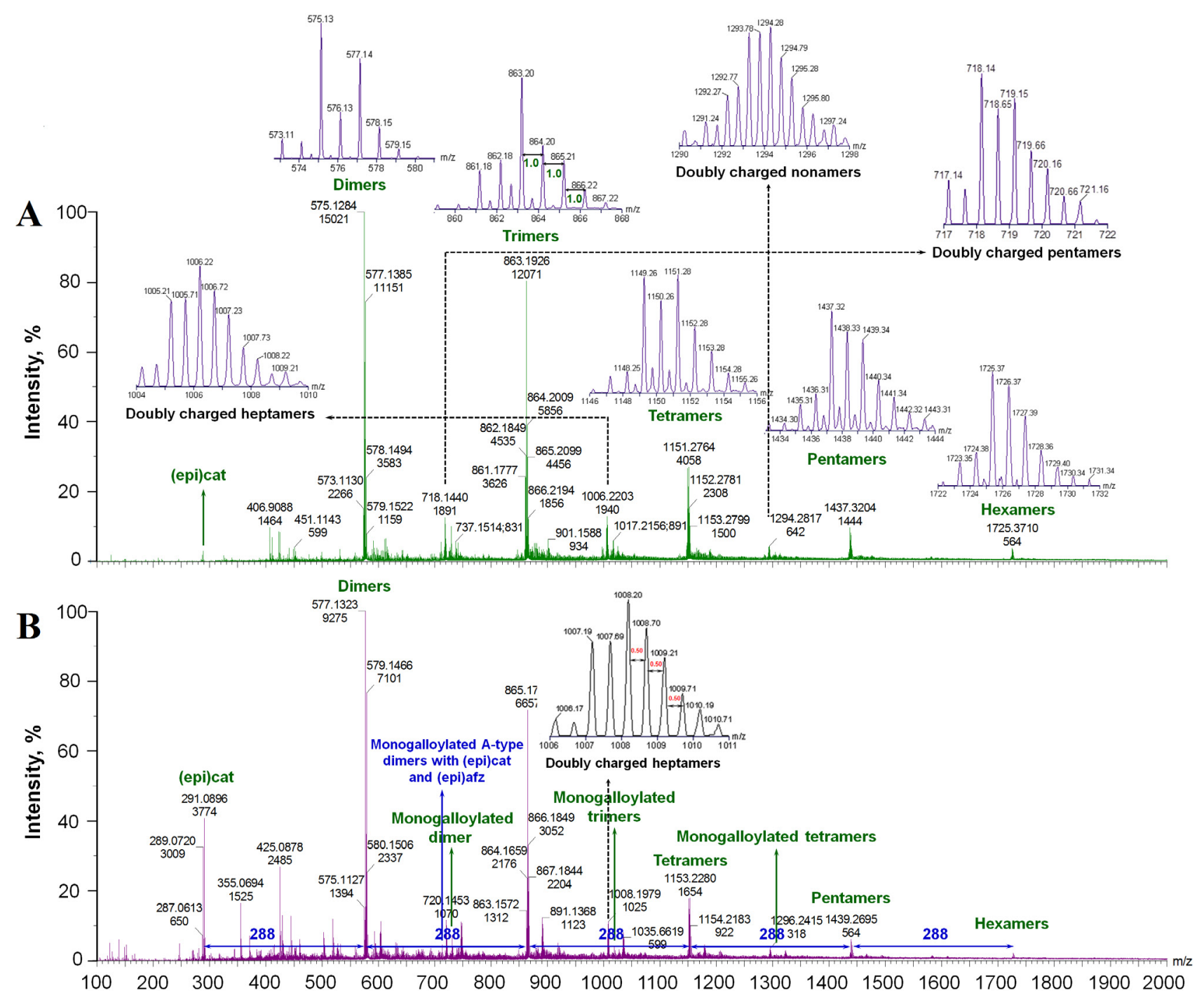

Figure 2: Direct-infusion ESI-Q-TOF-MS profiles of grape seed extract at positive and negative ion ESI modes.

A: GSE, ESI-; B: GSE, ESI+; "288" means the mass difference between the two closest PACs

Inserts in A are enlarged spectra in negative mode showing overlapped isotope patterns of proanthocyanidin dimers, trimers, tetramers, pentamers, and hexamers as well as doubly charged pentamers, heptamers and nonamers containing A- and B- types. Insert in B is an enlarged spectrum in positive mode showing overlapped isotope patterns of doubly charged heptamers containing A- and B- types

previous reports $[24,35,36,40]$. Trimers with one A-typelinkage couldbe assigned as (epi)cat-A-(epi)cat-(epi)cat or (epi)cat-(epi)cat-A-(epi)cat depending on the fragments: (epi)cat-A-(epi)cat-(epi)cat has fragement ions at $\mathrm{m} / \mathrm{z} 573$ and 289 via QM cleavages between middle and terminal units while (epi)cat-(epi)cat-A-(epi)cat has $\mathrm{m} / \mathrm{z} 575$ and 287 fragments via QM cleavages between top and middle units. In addition, both of them generate fragments at $\mathrm{m} / \mathrm{z} 737$ (loss of $126 \mathrm{Da}$ through HRF) and 711 (loss $152 \mathrm{Da}$ by RDA). Under ESI+ mode,the precursorions at $\mathrm{m} / \mathrm{z} 865^{*}$ and $863^{*}$ are PAC trimers with 1 and 2 A-type linkages. Based on their fragments in table 2, the $[\mathrm{M}+\mathrm{H}]^{+}$at $\mathrm{m} / \mathrm{z} 865^{*}$ might be (epi)cat-(epi)cat-A-(epi)cat [6].

PAC tetramers, pentamers and hexamers with 1 to 3 A-type linkages were detected under ESI- with the corresponding precusor ions at $\mathrm{m} / \mathrm{z} 1151,1149$ and 1147; 1439, 1437 and 1435; 1727, 1725 and 1723 (insert in Figure 2A). The main fragments of [M-H] ions at $\mathrm{m} / \mathrm{z} 1147,1149,1435,1437$ and 1725 are listed in table 2, which are generally produced from [M-H-152] (RDA), [M-H-(288) $]^{-}$ (progressively loss (epi)cat units) or loss water molecules. A portion of these precursor ions and possible isomers of tetramers and pentamers with one and two A-type linkages are mentioned previously in other foods such as peanuts and cranberry $[5,24,35,36]$.

Under ESI+ mode, tetramers, pentamers and hexamers with 1 and 2 A-type linkages were also detected in GSE with precursor ions at $\mathrm{m} / \mathrm{z} 1153^{\star}$ and $1151^{\star} ; 1441^{\star}$ and $1439^{\star} ; 1729^{\star}$ and $1727^{\star}$, respectively (Table 2). In addition, hexamers with three A-type linkages were detected in GSE at $\mathrm{m} / \mathrm{z} 1725^{*}$ under ESI+. Overall, with increasing the degree of polymerization, the detected amount of A-type PACs decreased. 
A

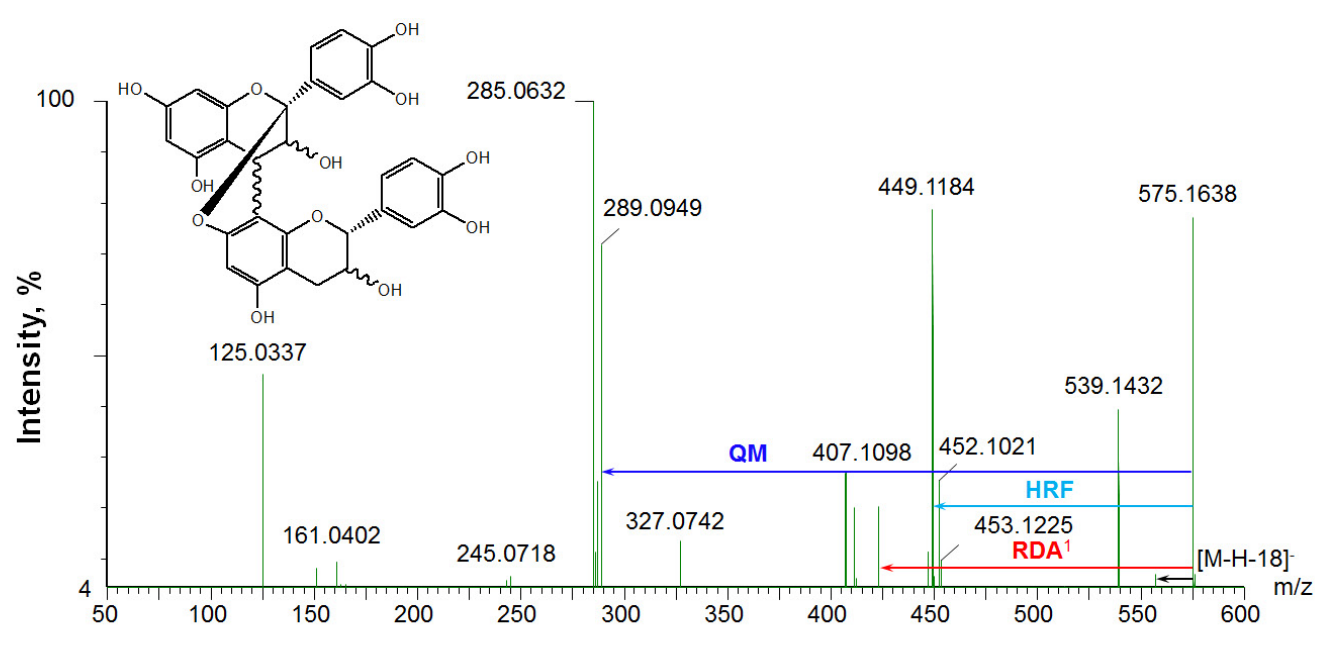

B

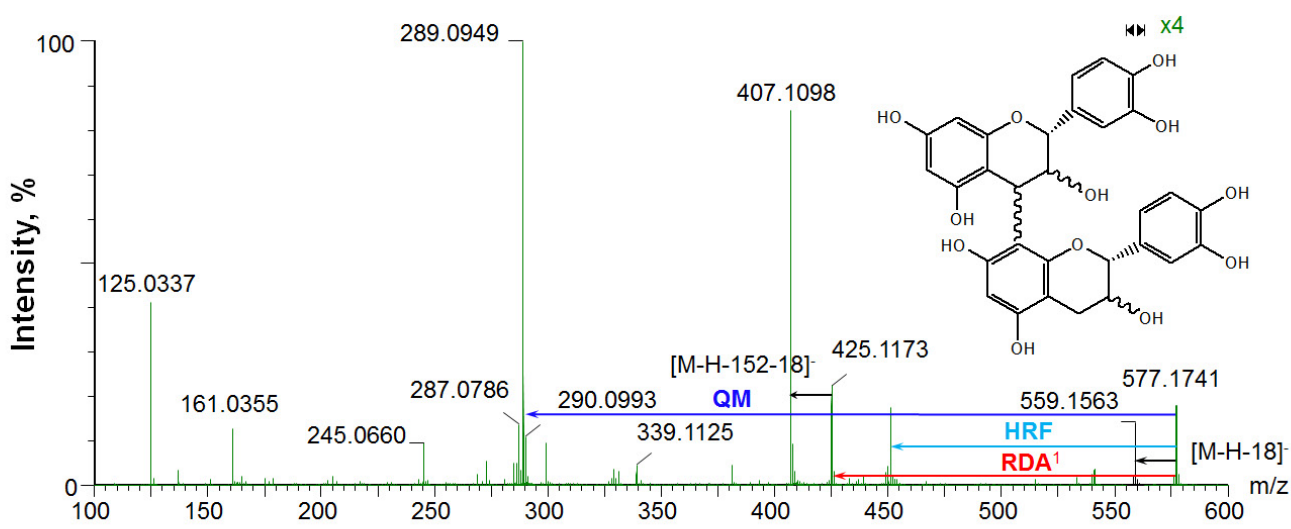

C

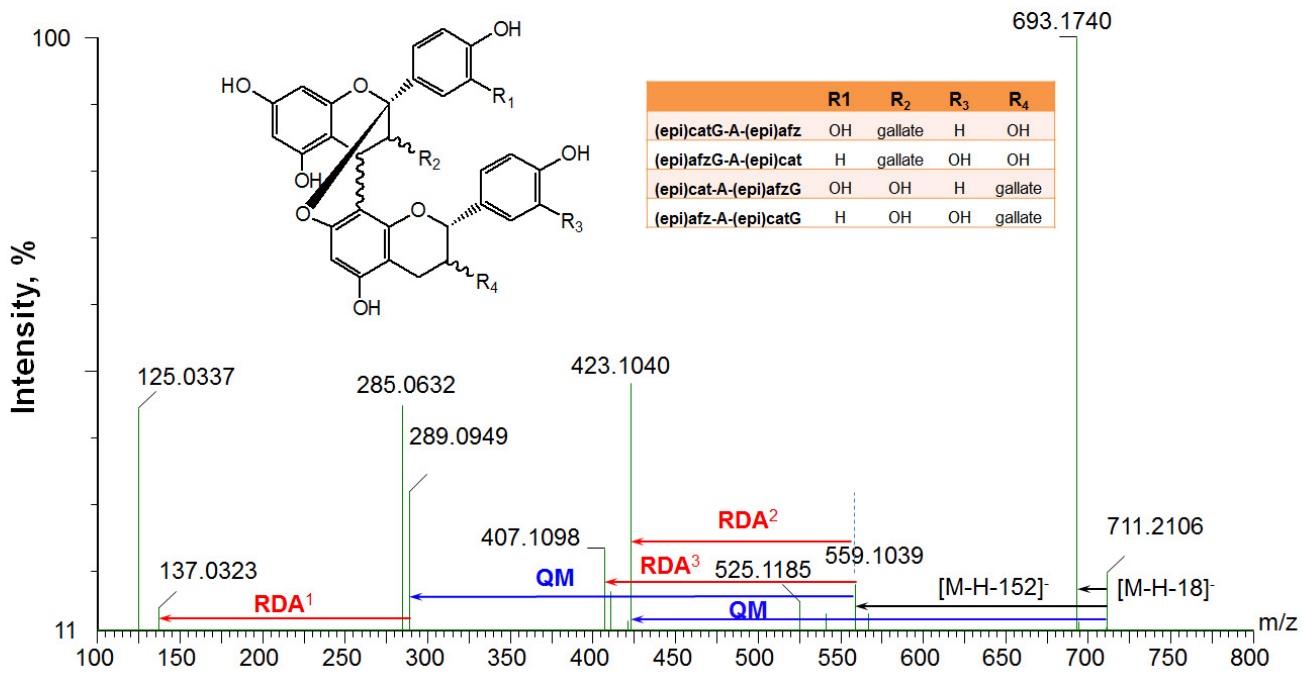

Figure 3: ESI-MS/MS spectra with possible main fragmentation pathways of $[\mathrm{M}-\mathrm{H}]-$ ions.

A: A-type dimer; B: B-type dimer; C: monogalloylated A-type dimers with (epi)cat and (epi)afz

RDA: Retro-Diels-Alder fission; QM: Quinone-Methide fission; HFR: Heterocyclic Ring Fission; [M-H-152]- in C: this fragment comes from two possible ways: 1) loss of one galloyl group, 2) via RDA if the terminal unit is (epi)cat. RDA1 means loss of 152 Da from precursor ions (A,B) or the fragment of m/z 289 (C); RDA2: this fragment generates from the fragment of $\mathrm{m} / \mathrm{z} 559$ (loss one galloyl group) through RDA if the terminal unit is or to become (epi)afz (lose 136 Da) (C); RDA3: this is from the fragment of $\mathrm{m} / \mathrm{z} 559$ (loss one galloyl group) through RDA if the terminal unit is or to become (epi)cat (lose 152 Da) (C), HRF, [M-H-126]-; QM, lose one (epi)catechin; " 4 " means the fragment of $\mathrm{m} / \mathrm{z} 559$ is zoomed in by 4 times 


\begin{tabular}{|c|c|c|c|c|c|c|}
\hline & \multicolumn{2}{|c|}{ GSE } & \multicolumn{2}{c|}{ RGPE } & \multicolumn{2}{c|}{ WGPE } \\
\hline & ESI- & ESI+ & ESI- & ESI+ & ESI- & ESI+ \\
\hline Monomer & $0.32 \pm 0.01$ & $13.05 \pm 0.34$ & $1.89 \pm 0.07$ & $26.72 \pm 0.32$ & $11.65 \pm 0.11$ & $36.93 \pm 0.34$ \\
\hline Dimer & $37.71 \pm 1.96$ & $41.46 \pm 1.07$ & $50.33 \pm 0.14$ & $45.28 \pm 0.40$ & $45.46 \pm 0.17$ & $38.57 \pm 0.58$ \\
\hline Trimer & $35.75 \pm 0.29$ & $29.63 \pm 1.19$ & $26.43 \pm 0.03$ & $16.63 \pm 0.25$ & $24.65 \pm 0.02$ & $8.99 \pm 0.06$ \\
\hline Tetramer & $20.24 \pm 0.22$ & $11.91 \pm 0.45$ & $12.54 \pm 0.34$ & $6.29 \pm 0.06$ & $12.64 \pm 0.39$ & $7.53 \pm 0.12$ \\
\hline Pentamer & $4.85 \pm 1.43$ & $3.30 \pm 0.11$ & $6.16 \pm 0.04$ & $4.02 \pm 0.13$ & $3.96 \pm 0.23$ & $5.23 \pm 0.36$ \\
\hline Oligomers & $98.56 \pm 0.60$ & $86.30 \pm 0.46$ & $95.46 \pm 0.14$ & $72.22 \pm 0.83$ & $86.71 \pm 0.07$ & $60.32 \pm 0.17$ \\
\hline
\end{tabular}

Table 3: Relative percentages of monomer and oligomers in total proanthocyanidins.

ESI+: Electrospray lonization at positive mode; ESI-: Electrospray lonization at negative mode; GSE: Grape Seed Extract; RGPE: Red Grape Pomace Extract; WGPE: White Grape Pomace Extract

Doubly charged A-type PACs: Doubly charged ([M-2H $\left.]^{2-}\right)$ A-type PACs were detected only in GSE (inserts in Figure 2). Under ESI-, $[\mathrm{M}-2 \mathrm{H}]^{2-}$ PAC pentamers with 1-3 A-type linkages (m/z 1439, 1437 and 1435 , respectively) were occurred at $\mathrm{m} / \mathrm{z} 719,718$ and 717 , respectively (insert in Figure 2A). [M-2H $]^{2-}$ heptamers with 1-3 A-type linkages (m/z 2015, 2013 and 2011, respectively) were detected at $\mathrm{m} / \mathrm{z}$ 1007,1006 and 1005, respectively under ESI- mode (insert in Figure 2A). Under ESI+, the double charged heptamers with 1-3 A-type linkages were at $\mathrm{m} / \mathrm{z} 1009^{\star}, 1008^{*}$ and $1007^{*}$ (insert in Figure $2 \mathrm{~B}$ ). $[\mathrm{M}-2 \mathrm{H}]^{2-} \mathrm{PAC}$ nonamers with 1-4 A-type linkages (m/z 2591, 2589, 2587, and 2585, respectively) were also detected respectively at $\mathrm{m} / \mathrm{z} 1295,1294,1293$ and 1292 (insert in Figure 2A). Up to now, there was no detailed report about doubly charged precursor ions in grapes, especially doubly charged A-type PACs, though doubly charged A-type PAC tetramers $(\mathrm{m} / \mathrm{z} 1149)$ and pentamers $(\mathrm{m} / \mathrm{z}$ 1439) were recently reported in dry-blanched peanut skins [24].

Some singly charged precursor ions overlaped with the doubly charged ones in some cases along with some unkown precusor ions with high intensities such as at $\mathrm{m} / \mathrm{z} 313,325,359$, and 439 in grape pomaces warrant future characterization.

Relative content of PACs analyzed by ESI Q-TOF MS: The relative content of monomeric and polymeric (epi)catechins were different under different ionization mode (Table 3). The percentage of monomeric (epi)catechin calculated from ESI+ mode was much higher than that from ESI- mode, but the relative content of oligomers from ESI- was generally higher than that from ESI+ (Table 3). Overall, oligomers were the major PACs in all grape extracts, dominant by dimers and trimers.

\section{Conclusion}

RGPE had higher content of phenolics, flavonoids and proanthocyanidins, and antioxidant activities than WGPE. The oligomers of (epi)catechin were the major PACs in all grape extracts studied. Monogalloylated dimers, trimers and tetramers were detected in GSE, RGPE and WGPE, while anthocyanins were detected only in RGPE and WGPE. The B-type PACs could be found in all grape extracts under both ESI-/+ mode, while A-type PACs were more detectable in GSE. Under ESI-, A-type dimer, single A-type linked PAC trimers and tetramers can be also detected in RGPE and WGPE in addtion to GSE. Of which, monogalloylated A-type dimers with (epi)cat and (epi)afz were detected in both GSE and WGPE for the first time.

\section{References}

1. Guendez R, Kallithraka S, Makris DP, Kefalas P (2005) An analytical survey of the polyphenols of seeds of varieties of grape (Vitis vinifera) cultivated in Greece: implications for exploitation as a source of value-added phytochemicals. Phytochem Anal 16: 17-23.

2. Lizarraga $D$, Vinardell MP, Noé V, van Delft JH, Alcarraz-Vizán G, et al. (2011) A lyophilized red grape pomace containing proanthocyanidin-rich dietary fiber induces genetic and metabolic alterations in colon mucosa of female C57BL/6J mice. J Nutr 141: 1597-1604.

3. Wang $\mathrm{H}$, Xue $\mathrm{Y}$, Zhang $\mathrm{H}$, Huang $\mathrm{Y}$, Yang G, et al. (2013) Dietary grape seed extract ameliorates symptoms of inflammatory bowel disease in IL10-deficient mice. Mol Nutr Food Res 57: 2253-2257.

4. Cheah KY, Bastian SE, Acott TM, Abimosleh SM, Lymn KA, et al. (2013) Grape seed extract reduces the severity of selected disease markers in the proximal colon of dextran sulphate sodium-induced colitis in rats. Dig Dis Sci 58: 970-977.

5. Gu L, Kelm MA, Hammerstone JF, Zhang Z, Beecher G, et al. (2003) Liquid chromatographic/electrospray ionization mass spectrometric studies of proanthocyanidins in foods. J Mass Spectrom 38: 1272-1280.

6. Passos CP, Cardoso SM, Domingues MRM, Domingues P, Silva CM, et al. (2007) Evidence for galloylated type-A procyanidins in grape seeds. Food Chemistry 105: 1457-1467.

7. Rockenbach II, Jungfer E, Ritter C, Santiago-Schübel B, Thiele B, et al. (2012) Characterization of flavan-3-ols in seeds of grape pomace by CE, HPLC-DAD-MS ${ }^{n}$ and LC-ESI-FTICR-MS. Food Research International 48: 848-855.

8. Howell AB, Reed JD, Krueger CG, Winterbottom R, Cunningham DG, et al. (2005) A-type cranberry proanthocyanidins and uropathogenic bacterial anti-adhesion activity. Phytochemistry 66: 2281-2291.

9. Yokota K, Kimura H, Ogawa S, Akihiro T (2013) Analysis of A-Type and B-Type Highly Polymeric Proanthocyanidins and Their Biological Activities as Nutraceuticals. Journal of Chemistry: 1-7.

10. Singleton VL, Rossi JA (1965) Colorimetry of total phenolics with phosphomolybdic-phosphotungstic acid reagents. Am J Enol Vitic 16: 144-158.

11. Dame CJ, Chichester CO, Marsh GL (1957) Studies of processed all-green asparagus: I. quantitative analysis of soluble compounds with respect to strain and harvest variables, and their distribution within the asparagus spear. Journal of Food Science 22: 658-672.

12. Garcia-Parrilla MC, Heredia FJ, Troncoso AM, González GA (1997) Spectrophotometric determination of total procyanidins in wine vinegars. Talanta 44: 119-123.

13. Brand-Williams W, Cuvelier ME, Berset C (1995) Use of a free radical method to evaluate antioxidant activity. LWT - Food Science and Technology 28 : 25-30.

14. González-Centeno MR, Jourdes M, Femenia A, Simal S, Rossello C, et al. (2013) Characterization of polyphenols and antioxidant potential of white grape pomace byproducts (Vitis vinifera L.). J Agric Food Chem 61: 1157911587. 
15. Hogan S, Canning C, Sun S, Sun X, Kadouh H, et al. (2011) Dietary supplementation of grape skin extract improves glycemia and inflammation in diet-induced obese mice fed a Western high fat diet. J Agric Food Chem 59 3035-3041.

16. Rockenbach II, Rodrigues E, Gonzaga LV, Caliari V, Genovese MI, et al. (2011) Phenolic compounds content and antioxidant activity in pomace from selected red grapes (Vitis vinifera L. and Vitis labrusca L.) widely produced in Brazil. Food Chemistry 127: 174-179.

17. Hogan S, Zhang L, Li J, Sun S, Canning C, et al. (2010) Antioxidant rich grape pomace extract suppresses postprandial hyperglycemia in diabetic mice by specifically inhibiting alpha-glucosidase. Nutr Metab (Lond) 7: 71.

18. Parry JW, Li H, Liu JR, Zhou K, Zhang L, et al. (2011) Antioxidant activity, antiproliferation of colon cancer cells, and chemical composition of grape pomace. Food and Nutrition Sciences 2: 530-540.

19. Yemis O, Bakkalbasi E, Artik N (2008) Antioxidative activities of grape (Vitis vinifera) seed extracts obtained from different varieties grown in Turkey. International Journal of Food Science and Technology 43: 154-159.

20. Li HJ, Deinzer ML (2007) Tandem mass spectrometry for sequencing proanthocyanidins. Anal Chem 79: 1739-1748.

21. Li HJ, Deinzer ML (2008) The mass spectral analysis of isolated hops A-type proanthocyanidins by electrospray ionization tandem mass spectrometry. $J$ Mass Spectrom 43: 1353-1363.

22. Biasoto ACT, Catharino RR, Sanvido GB, Eberlin MN, da Silva MAAP (2010) Flavour characterization of red wines by descriptive analysis and ESI mass spectrometry. Food Quality and Preference 21: 755-762.

23. Cottica SM, de Morais DR, Rotta EM, Sargi SC, Silva FLN, et al. (2013) Effects of grape processing on antioxidant capacity and ESI-MS fingerprints of grape products. Journal of Food Science and Engineering 3: 341-348.

24. Ma Y, Kosińska-Cagnazzo A, Kerr WL, Amarowicz R, Swanson RB, et al. (2014) Separation and characterization of soluble esterified and glycoside-bound phenolic compounds in dry-blanched peanut skins by liquid chromatography-electrospray ionization mass spectrometry. J Agric Food Chem 62: $11488-11504$.

25. Cantos E, Espín JC, Tomás-Barberán FA (2002) Varietal differences among the polyphenol profiles of seven table grape cultivars studied by LC-DAD-MSMS. J Agric Food Chem 50: 5691-5696.

26. Kammerer D, Claus A, Carle R, Schieber A (2004) Polyphenol screening of pomace from red and white grape varieties (Vitis vinifera L.) by HPLC-DADMS/MS. J Agric Food Chem 52: 4360-4367.

27. Wang H, Race EJ, Shrikhande AJ (2003) Characterization of anthocyanins in grape juices by ion trap liquid chromatography-mass spectrometry. J Agric Food Chem 51: 1839-1844.

28. Xu Y, Simon JE, Welch C, Wightman JD, Ferruzzi MG, et al. (2011) Survey of polyphenol constituents in grapes and grape-derived products. J Agric Food Chem 59: 10586-10593.
29. Sandhu AK, Gu L (2010) Antioxidant capacity, phenolic content, and profiling of phenolic compounds in the seeds, skin, and pulp of Vitis rotundifolia (Muscadine Grapes) As determined by HPLC-DAD-ESI-MS(n). J Agric Food Chem 58: 4681-4692.

30. Crupi P, Coletta A, Anna Milella R, Perniola R, Gasparro M, et al. (2012) HPLC-DAD-ESI-MS analysis of flavonoid compounds in 5 seedless table grapes grown in Apulian Region. J Food Sci 77: 174-181.

31. Tala VR, Candida da Silva V, Rodrigues CM, Nkengfack AE, dos Santos LC, et al. (2013) Characterization of proanthocyanidins from Parkia biglobosa (Jacq.) G. Don. (Fabaceae) by flow injection analysis-electrospray ionization ion trap tandem mass spectrometry and liquid chromatography/electrospray ionization mass spectrometry. Molecules 18: 2803-2820.

32. Montero L, Herrero M, Prodanov M, Ibáñez E, Cifuentes A (2013) Characterization of grape seed procyanidins by comprehensive two-dimensional hydrophilic interaction $x$ reversed phase liquid chromatography coupled to diode array detection and tandem mass spectrometry. Anal Bioanal Chem 405: 4627-4638.

33. Hayasaka Y, Waters EJ, Cheynier V, Herderich MJ, Vidal S (2003) Characterization of proanthocyanidins in grape seeds using electrospray mass spectrometry. Rapid Commun Mass Spectrom 17: 9-16.

34. Oliveria J, da Silva MA, Parola AJ, Mateus N, Brás NF, et al. (2013) Structural characterization of a A-type linked trimeric anthocyanin derived pigment occurring in young Port wine. Food Chem 141: 1987-1996.

35. Appeldoorn MM, Vincken JP, Sanders M, Hollman PC, Gruppen H (2009) Combined normal-phase and reversed-phase liquid chromatography/ESI-MS as a tool to determine the molecular diversity of A-type procyanidins in peanut skins. J Agric Food Chem 57: 6007-6013.

36. Lin LZ, Sun J, Chen P, Monagas MJ, Harnly JM (2014) UHPLC-PDA-ESI/ HRMSn profiling method to identify and quantify oligomeric proanthocyanidins in plant products. J Agric Food Chem 62: 9387-9400.

37. Ogawa S, Kimura H, Niimi A, Katsube T, Jisaka M, et al. (2008) Fractionation and structural characterization of polyphenolic antioxidants from seed shells of Japanese horse chestnut (Aesculus turbinata BLUME). J Agric Food Chem 56: 12046-12051.

38. Jaiswal R, Jayasinghe L, Kuhnert N (2012) Identification and characterization of proanthocyanidins of 16 members of the Rhododendron genus (Ericaceae) by tandem LC-MS. J Mass Spectrom 47: 502-515.

39. Zhu QY, Hammerstone JF, Lazarus SA, Schmitz HH, Keen CL (2003) Stabilizing effect of ascorbic acid on flavan-3-ols and dimeric procyanidins from cocoa. J Agric Food Chem 51: 828-833.

40. Garrett R, Romanos MTV, Borges RM, Santos MG, Rocha L, et al. (2012) Antiherpetic activity of a flavonoid fraction from Ocotea notata leaves. Rev bras farmacogn 22: 306-313. 